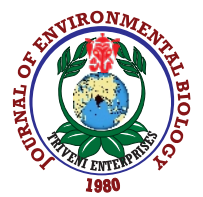

\title{
Synthesis, characterization of blue fluorescent carbon nanoparticle and its in-vitro toxicity evaluation
}

\author{
K.S. Kumar', P.K. Gayathri' ${ }^{1 *}$, H. Khandelwal ${ }^{2}$ and K.K. Prashanth ${ }^{2}$ \\ 'Department of Chemical Engineering, SSN Engineering College, Kalavakkam-603 110, India \\ ${ }^{2}$ Department of Biotechnology, Vel Tech High Tech Dr. Rangarajan Dr. Sakunthala Engineering College, Avadi-600 062, India \\ *Corresponding Author Email : gayathri.kothandaraman@gmail.com
}

\begin{tabular}{lll}
\hline Paper received: 09.12.2019 & Revised received: 20.03.2020 Accepted:03.07.2020
\end{tabular}

\section{Abstract}

Aim: The present study aimed to synthesize and characterize carbon nanoparticles (CNPs) from a new precursor and to evaluate its effect on the viability of healthy seeds and cell lines.

Methodology: The CNPs were synthesized by acid assisted carbonization of carbohydrate and its characteristics such as size, fluorescence property and purity of samples were evaluated for confirmation. Cytotoxic studies were performed in MCF 10a cell lines using 3-4,5-dimethylthiazol2,5-diphenyltetrazolium bromide (MTT) assay whereas the phytotoxic assay was conducted by using scallions and Indian pearl millet.

Results: Transmission electron microscope (TEM) results showed that nano-sized CNPs [10$40 \mathrm{~nm}$ ] were synthesized and its characteristic peaks were observed in Ultraviolet (UV) and fluorescence spectrum. The Energy Dispersive Xray (EDAX) analysis confirmed the presence of carbon and oxygen without any impurities. It was non-cytotoxic for healthy MCF 10a cell lines and did not affect the germination rate of Indian pearl millets at higher concentrations. In both toxicity
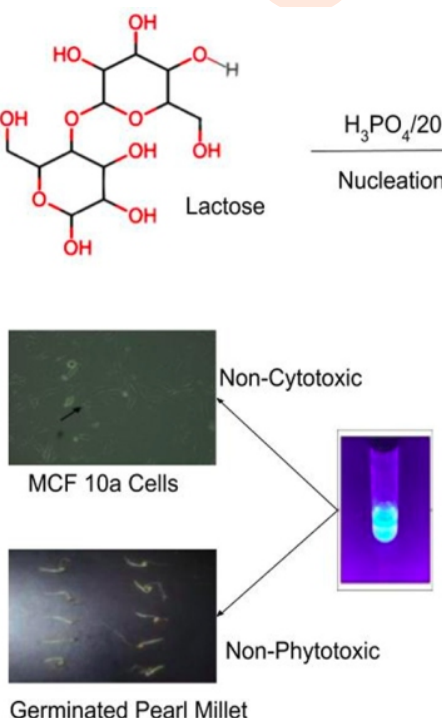

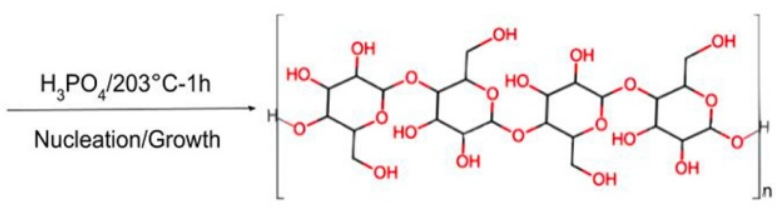
$203^{\circ} \mathrm{C} / 1 \mathrm{~h} \quad$ Carbonization

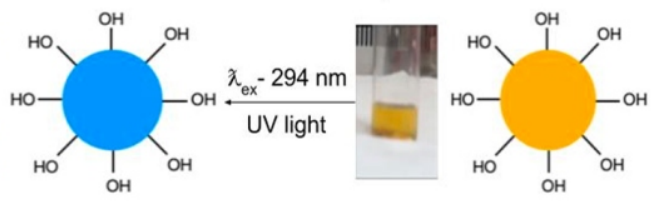

Normal light studies, the viability of cells and plants was $>95 \%$ even after exposure to a high concentration of CNPs.

Interpretation: The non-cytotoxic and non-phytotoxic nature of CNPs implies that it does not affect the growth of plants, seedling germination and healthy cell lines. It can be considered as a compatible and safe material for MCF 10a cell lines and plants.

Key words: Carbon nanoparticles, Fluorescence study, MCF 10a cell lines, MTT assay, Pearl millet

How to cite : Kumar, K.S., P.K. Gayathri, H. Khandelwal and K.K. Prashanth: Synthesis, characterization of blue fluorescent carbon nanoparticle and its in-vitro toxicity evaluation. J. Environ. Biol., 41, 1442-1449 (2020). 


\section{Introduction}

The development of several nanoparticles for biomedical applications has become a recent trend in industries and academia due to increased surface to volume ratio which leads to alterations in the physico-chemical and optical properties of nanomaterial during synthesis from bulk material (Sezer and Cevher, 2011; Gatoo et al., 2014). Nanomaterials with better biocompatibility, high aqueous solubility and photoluminescence are utilized widely in the field of biomedical applications like imaging, drug delivery carriers, labeling of cells and targeted delivery of drugs (Shi et al., 2014; Choi and Sun, 2011; Song et al., 2014). The resources from which the nanomaterials are synthesized is another area of interest, where the availability of source becomes a great challenge. The short and long term effects of metal nanoparticles on humans and the environment can be questioned during its potential use in biomedical applications. Therefore, the use of green source and synthesis methods becomes crucial to waive off the toxicity and origin of nanoparticles. There are several reports which suggest CNPs synthesis from natural and synthetic sources. Zhang et al. (2018) divided the natural resources as either plant or animal-derived. The plant-derived resources include biomass from plants and trees whereas some of the animal-derived sources include milk, bee's pollen, eggs and honey (Wu et al., 2013). The synthetic sources utilized by earlier researchers are organic molecules like simple sugars, amino acids, polymers, folic acid (Kim et al., 2014, Liu et al., 2018; Zhang et al., 2018) and food products like caramelized bread, jaggery, corn flakes and biscuit (Sk et al., 2012) and orange juice (Sahu et al., 2012).

It is pertinent to mention that the primary ingredients in most of these precursors are sugars. Hence, the prime motive of the study is to use a new source for synthesis of carbon nanoparticles. There are two approaches to synthesize CNPs, which include top-down and bottom-up approaches. The topdown approach involves breaking complex macro-materials whereas the bottom-up approach involves the growing of simple materials (Pengli et al., 2016; Wu et al., 2017; Anirudh and Joydeep, 2019). In this study, a bottom-up approach was adopted to synthesize CNPs from a monomer with minor variations. The desirable features of a nanomaterial are the ease of manufacturing, stability over a long period, biocompatibility and bioavailability. The small size of nanomaterial may fulfill the bioavailability and stability criteria whereas biocompatibility becomes a huge debate (Choi and Sun, 2011; Zhang et al., 2018). Hence, it has become imperative to study and understand the biocompatibility of nanomaterials before going for an application. Since the main aim was to develop carbon nanoparticles for biological applications, the degree of toxicity of these CNPS needs to be determined.

The production of nanoparticles on a larger scale requires two important processing steps such as upstream processing and downstream processing. The upstream processing includes material synthesis, formulation and quality check whereas the downstream processing includes filtration, various purification and separation processes and drying. The downstream processing separates and discards the nanoparticles that are beyond the cutoff particle size and desirable surface characteristics. If the discarded nanoparticles directly accumulate in the soil, it can affect the growth of healthy plants and suppress seed germination.

Several inconsistent reports were published on the toxicity of nanoparticles on human cells. For example, VillanuevaFlores et al. (2020) suggested that nanoparticles of size less than $1.5 \mathrm{~nm}$ can interact with proteins or interfere with cellular channels and react causing inflammatory response or oxidative stress. On the contrary, Kokorina et al. (2017) reviewed the biomedical application of non-cytotoxic carbon nanoparticles synthesized from different sources with variable size range between 0.5 and $120 \mathrm{~nm}$. Therefore, information on the interaction between nanoparticles and cell membrane is still incomplete. Since the synthesized carbon nanoparticles in this study will be used in biological-based applications, cytotoxicity evaluation was performed on healthy MCF 10a epithelial cell lines because CNPs interact with both healthy and target cells, and the free form of CNPs should not affect healthy cells. The objective of the present study was to synthesize carbon nanoparticles (CNPs) and to evaluate its effect on the growth of plants, seed germination and viability of healthy cell lines.

\section{Materials and Methods}

Synthesis and characterization of CNPs: An acid assisted carbonization of monomer was utilized in this study with minor variations (Mora and Wood, 1958). Briefly, $1 \mathrm{~g}$ of lactose was mixed with $3 \%$ (v/v) orthophosphoric acid-water solution to obtain a final $\mathrm{pH}$ value of 3.0-5.0 and was heated in a hot air oven $\left(203^{\circ} \mathrm{C}\right)$ for $2 \mathrm{hr}$ with intermittent mixing. The final sample was dissolved in water, precipitated with 10 times volume of ice-cold ethanol and centrifuged at $10000 \mathrm{rpm}$ for $15 \mathrm{~min}$. It was dialyzed (MW cutoff - $3.5 \mathrm{kDa}$ ) in distilled water for $24 \mathrm{hr}$ and freeze-dried at $4^{\circ} \mathrm{C}$ for $12 \mathrm{hr}$. The freeze-dried CNPs were stored in an airtight container until further use. The UV absorption spectrum of CNPS was measured between 190-800 nm using Jasco spectrophotometer (Jasco V-650 series) and the elemental composition of sample was determined using EDAX genesis spectroscopy. For TEM imaging, the sample was prepared by drop-casting the sample $(5 \mu \mathrm{l})$ on a copper grid followed by airdrying, which was observed under a JEOL 3010 instrument. The fluorescent property was determined by dissolving the freezedried sample in distilled water and exposing to a fluorescent spectrophotometer (RF-6000 Shimadzu series) at an excitation wavelength between $270-300 \mathrm{~nm}$ with $600 \mathrm{~nm}$ min-1 scan speed and UV transilluminator ( $312 \mathrm{~nm})$.

In-vitro phytotoxicity study: The phytotoxicity of CNPs was determined by root-tip treatment and seedling emergence technique with slight modification (Curtis, 1961). In the root-tip treatment, the roots of scallions were trimmed from the shoot and 
immersed in a glass of water. An increasing concentration of CNPs ( 0.2 to $\left.1.0 \mathrm{mg} \mathrm{ml}^{-1}\right)$ was added to water and maintained in an environment with adequate sunlight. The growth of new leaves and viability of scallion roots were observed for 15 days. Distilled water without CNPs served as control. After the study period, the scallions were washed several times with water and were dissected to isolate the inner rinds. The rinds were then washed with water, and analyzed under Olympus BX-51 inverted fluorescent microscope. In the seedling emergence technique, the Indian pearl millets were sterilized in 3\% hydrogen peroxide and rinsed in distilled water. Seeds were soaked in $20 \mathrm{ml}$ of CNP solution $\left(0.2\right.$ to $\left.1.0 \mathrm{mg} \mathrm{ml}^{-1}\right)$ for $3 \mathrm{hr}$. The soaked seeds were placed on moist tissue paper placed in a chamber at $25^{\circ} \mathrm{C}$ and 5 $\mathrm{ml}$ of CNP solution was added over the tissue paper every day. After one week, the germination percentage of all seeds was calculated by visual inspection for the presence of shoot and root. In this assay, water was taken as negative control, while $\mathrm{AgNO}_{3}$ was taken as positive control.

In-vitro cytotoxic study: The cytotoxicity of CNPs on MCF10a cell line was assessed by MTT assay (Mosmann, 1983). The cells were cultured in MEM supplemented with 10\% FBS (Fetal Bovine Serum), $1 \%$ penicillin, and $1 \%$ streptomycin in a humidified atmosphere of $5 \% \mathrm{CO}_{2}$ at $37^{\circ} \mathrm{C}$. Cells $\left(1 \times 10^{5}\right.$ per well) were plated in $1 \mathrm{ml}$ of medium per well in 24-well plates and incubated in the presence of various concentrations of CNPs $\left(0.2\right.$ to $\left.1.0 \mathrm{mg} \mathrm{ml}^{-1}\right)$ in $0.1 \%$ DMSO for $24 \mathrm{hr}$ at $37^{\circ} \mathrm{C}$. The wells were washed with phosphate-buffered saline ( $\mathrm{pH} 7.4)$ and treated with freshly prepared MTT $\left(5 \mathrm{mg} \mathrm{ml}^{-1}\right)$. After $24 \mathrm{hr}$ incubation, viable cells were determined by the absorbance at $570 \mathrm{~nm}$ using a UV-Spectrophotometer. The experiment was conducted in triplicate and wells without CNPs were considered as blanks. The effect of samples on the proliferation of MCF10a cell lines was expressed as \% MTT reduction.

Statistical Analysis: Statistical analysis was performed by Oneway ANOVA ( $p \leq 0.05)$ and all data are presented as mean $\pm S D$ (standard deviation).

\section{Results and Discussion}

Sustainable carbon materials can be produced from carbohydrates by heating at high temperatures using hydrothermal treatment. Three process parameters important for the synthesis of carbon nanoparticles from lactose are reaction time, $\mathrm{pH}$ of the reaction solution and reaction temperature (Zhang et al., 2016). According to Lamer and Dinegar (1950), rapid nucleation of precursor molecules and stable growth of nucleated core fused with precipitation produced well-dispersed particles with narrow particle size distribution. Rapid nucleation from supersaturated colorless lactose solution was achieved by maintaining the oven temperature above the melting point of lactose $\left(>202.8^{\circ} \mathrm{C}\right)$. The growth of nucleated carbon core was initiated by the condensation reaction catalyzed by orthophosphoric acid wherein condensation produced lactose molecules on the pre-formed nuclei. When growth attained the saturation level after the reaction time of $2 \mathrm{hr}$, the solution turned viscous and dark brown which was then precipitated with ethanol to obtain well-dispersed carbon nanoparticles of controlled size. The reaction time above $2 \mathrm{hr}$ did not demonstrate any difference in the level of product recovery.

The synthesized CNPs showed good absorption and emission properties. The shoulder peak at $274 \mathrm{~nm}$ in the UV spectrum (Fig. 1A) corresponds to the formation of $-\mathrm{C}=\mathrm{C}$ - bond depicting $\Pi-\Pi^{*}$ electron transition (Peng and Travas-Sejdic,

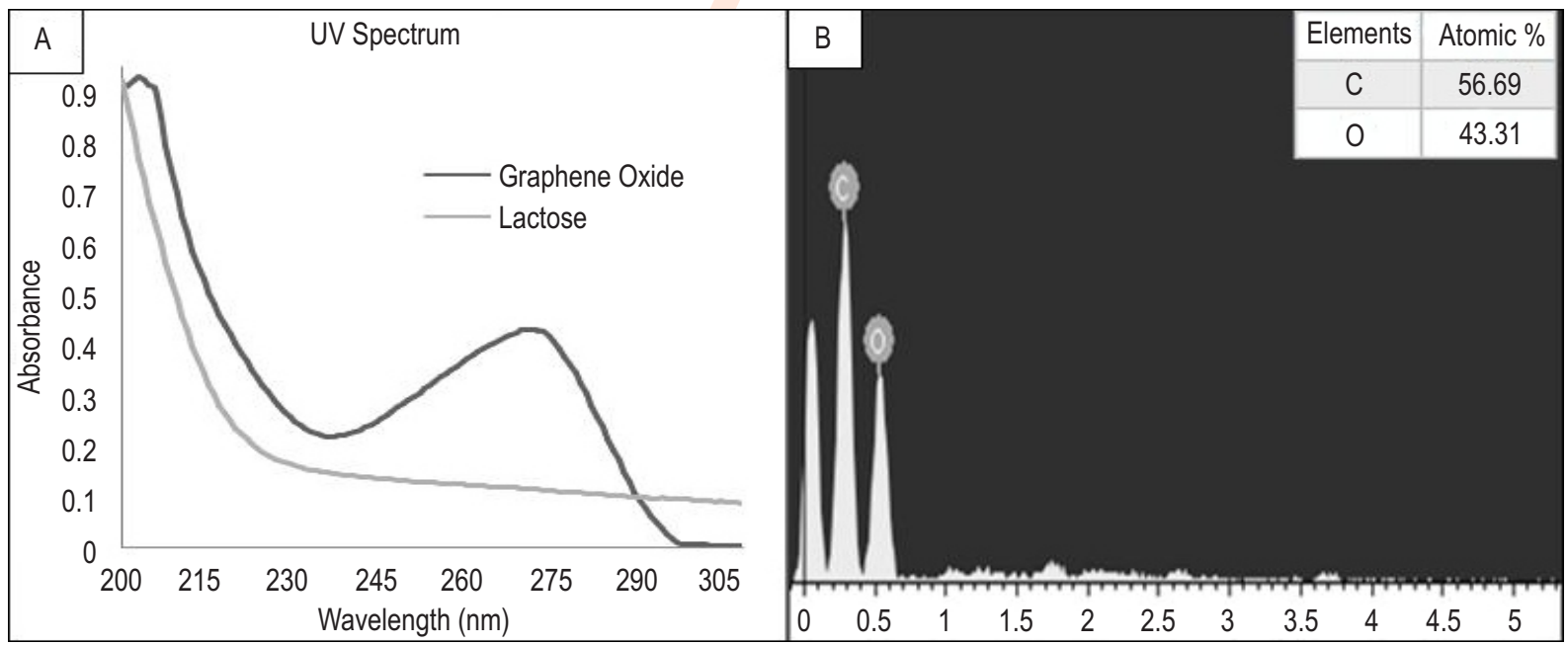

Fig. 1: (A) UV-Vis spectrum of CNPs showing a maximum absorption at $203 \mathrm{~nm}$ with a shoulder at $274 \mathrm{~nm}(2 \mathrm{hr})$; (B) EDAX spectrum showing the presence of only carbon (56.69\%) and oxygen (43.31\%) ions. 


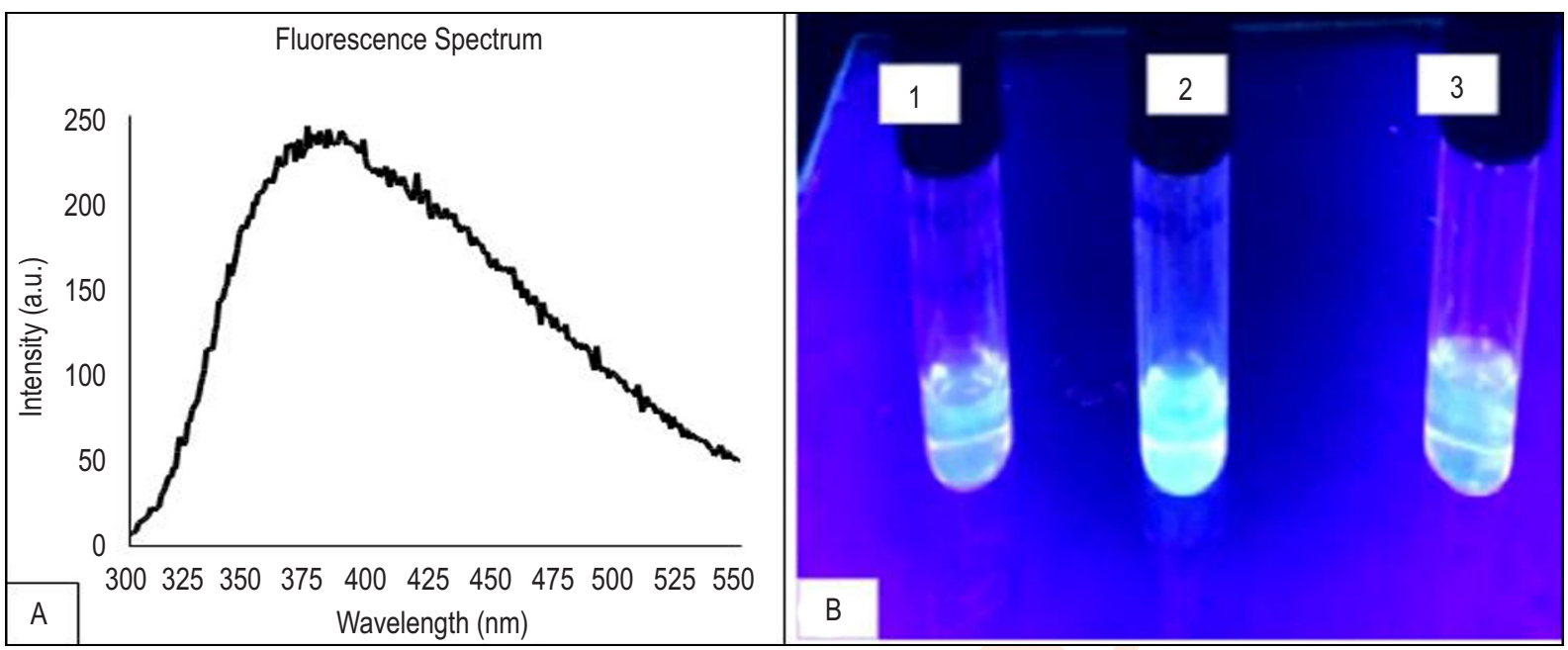

Fig. 2: (A) Fluorescence spectrum showing a maximum emission ( $\lambda$ em) at $383.6 \mathrm{~nm}$ wherein the excitation wavelength ( $\lambda$ ex) was $294 \mathrm{~nm}$. (B) Samples observed under UV transilluminator ( $312 \mathrm{~nm}$ ) wherein distilled water (1) and lactose solution (2) did not fluoresce whereas the CNPs containing solution (3) showed blue fluorescence that corresponds to the fluorescence emission spectrum (A).

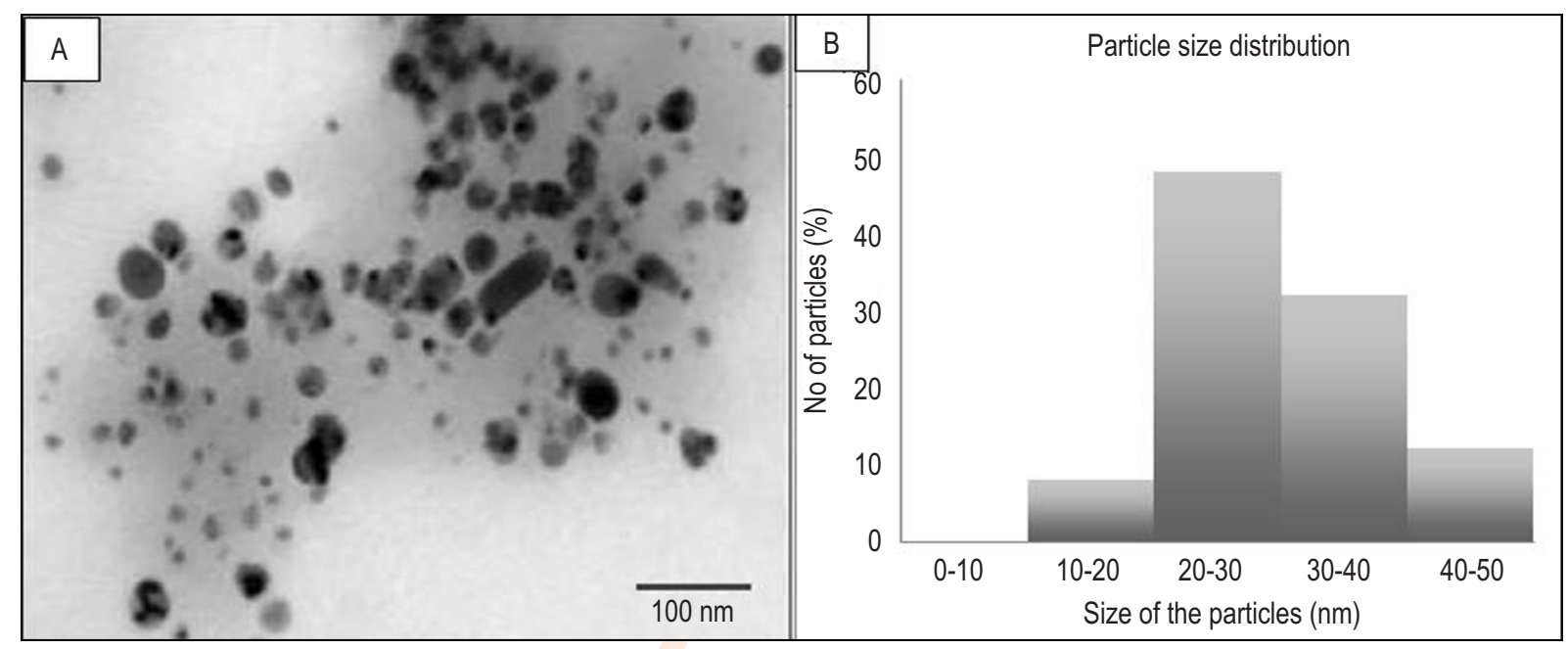

Fig. 3: (A) TEM image of the CNPs synthesized from lactose showing good dispersion in the medium. (B) Particle size analysis of the CNPs confirming the size distribution range of $10-50 \mathrm{~nm}$ with an average particle size of $20 \pm 2.5 \mathrm{~nm}$.

2009). This bond formation was due to the addition of lactose molecules during the growth phase of nanoparticle synthesis. The EDAX spectrum (Fig. 1B) confirmed the presence of carbon and oxygen ions and there were no additional peaks. This is attributed to simple purification steps like precipitation, moderate centrifugation and dialysis that played a decisive role to maintain the purity of CNPs. The range of excitation wavelength for fluorescence study was decided based on the absorbance peak in the UV-Vis spectrum (Wu et al., 2017). It is a well-known fact that the excitation wavelength was similar to absorption wavelength $(274 \mathrm{~nm})$, hence, the range of excitation wavelength was selected between $250-400 \mathrm{~nm}$. It was observed that the fluorescent spectrum (Fig. 2A) of dissolved sample showed a maximum emission peak $\left(\lambda_{\mathrm{em}}\right)$ at $383.6 \mathrm{~nm}$ at an excitation wavelength of $294 \mathrm{~nm}$. The spectrum indicated that the peak of emission wavelength $\left(\lambda_{\mathrm{em}}\right)$ had been shifted to longer wavelength $(383.6 \mathrm{~nm})$ from the excitation wavelength $(294 \mathrm{~nm})$ which complies with the concept of Stokes fluorescence shift. Since nanoparticles showed excitation in the UV wavelength range, the transparent CNP solution was observed in the UV transilluminator along with the negative control (distilled water) and lactose solution before heating. The CNPs containing solution alone 


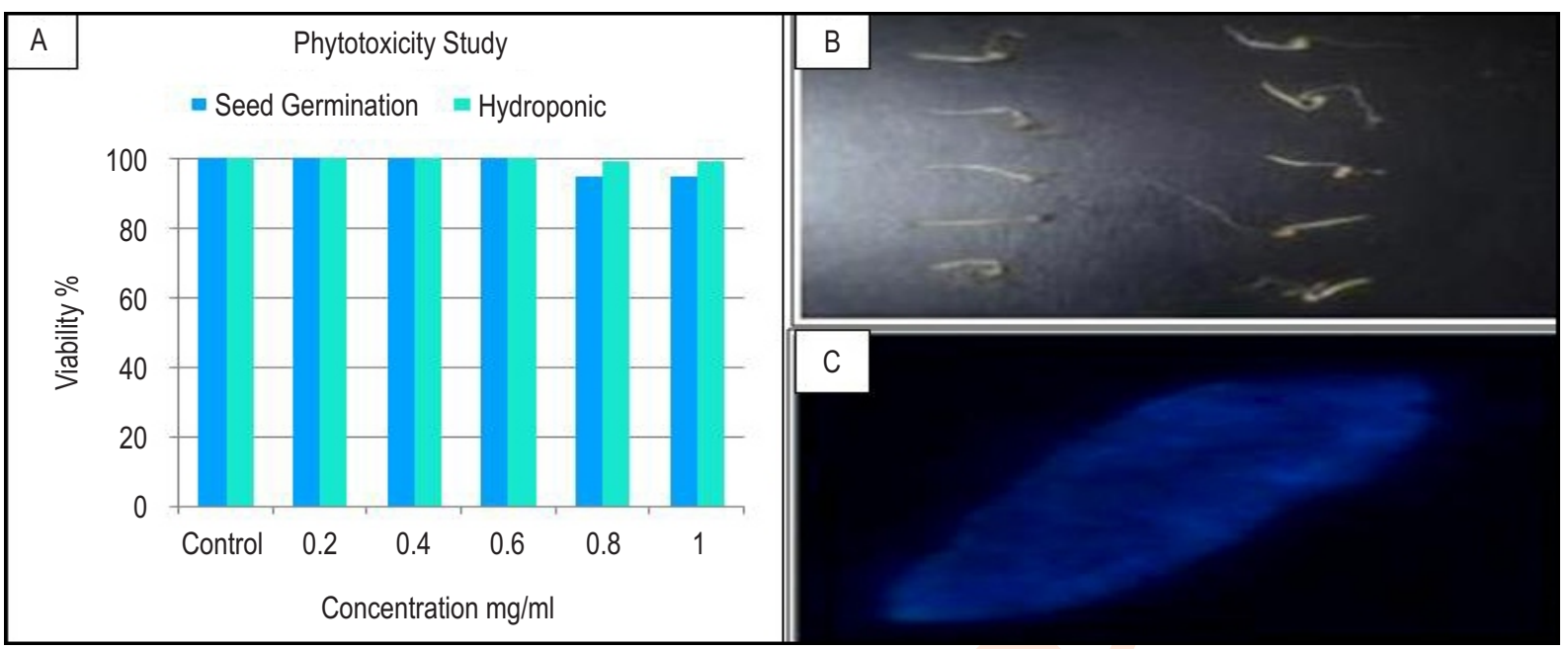

Fig. 4: (A) Comparative phytotoxicity analysis based on seed germination and hydroponic technique showing very minimal toxicity $(<2 \%)$ at the highest concentration of $1 \mathrm{mg} \mathrm{ml}^{-1}$. (B) One batch of germinated Indian pearl millet after exposure to $0.6 \mathrm{mg} / \mathrm{ml} \mathrm{CNPs} \mathrm{solution}$. (C) Observation of blue fluorescence in the isolated rind of scallion excited with a U-MWB band pass filter.

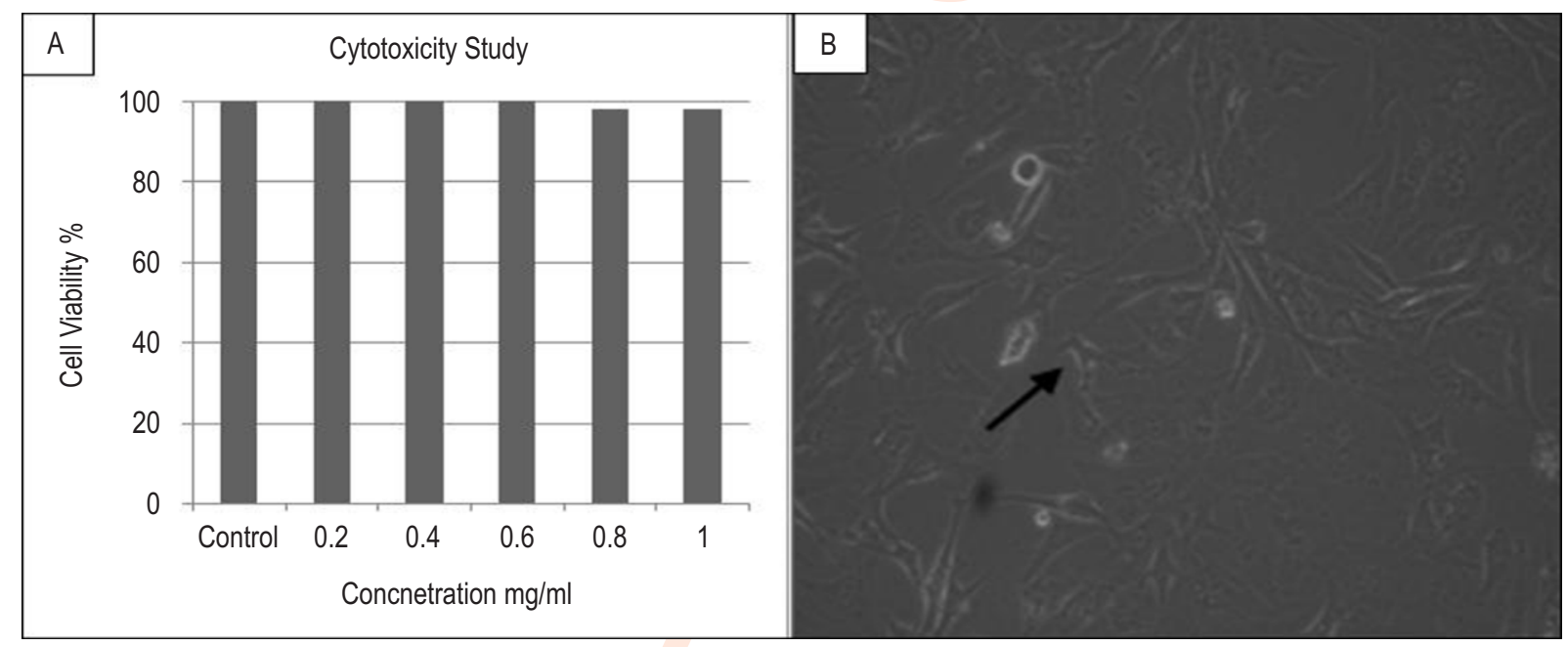

Fig. 5: (A) Results of Cytotoxicity assay showing maximum viability of MCF 10a cell lines in the concentration range of 0.2-1 mg ml ${ }^{-1}$. (B) Microscopic image of MCF 10a cells exposed to CNPs at $1.0 \mathrm{mg} \mathrm{ml}^{-1}$ showing only slight shrinkage in the cell structure (arrow).

emitted blue fluorescence whereas the other two samples showed no fluorescence (Fig. 2B).

To determine whether controlled particle size and dispersion of carbon nanoparticles were achieved as per the discussion above, characterization studies like TEM imaging and PS analysis were performed. As per TEM imaging (Fig. 3A), the CNPs showed good dispersion in the medium and the size of the particles analyzed using Image software ranged between 10-50 $\mathrm{nm}$. The size matched with the PSA results (Fig. 3B) showing an average particle size of about $20 \pm 2.5 \mathrm{~nm}$ and almost $75 \%$ of the particle size were $20 \mathrm{~nm}$. This is the first study giving insight on the use of lactose as precursor in the acid assisted synthesis of fluorescent CNPs and the characterization results are interpreted with previous literature. Md SK et al. (2012) and Haifang Liu et al. (2018) reported observation of dual peaks in UV spectrum between 200 and $350 \mathrm{~nm}$ and good mono dispersion in the TEM images for fluorescent carbon nanoparticles synthesized from folic acid and jaggery, respectively. The average particle size of CNPs synthesized from jaggery and folic acid was $20.3 \mathrm{~nm}$ and $5.4 \mathrm{~nm}$, that suggested it was dependent on the reaction time (5$10 \mathrm{~min}$ and $6 \mathrm{hr}$, respectively). On comparing, our current results 
are in good agreement with this discussion for fluorescent CNPs. The prior art on fluorescent carbon dots revealed that the luminescent property depends on the dispersion state of the particles, the number of particles excited at a specific wavelength, surface characteristics and size of nanoparticle. Well dispersed nanoparticles in neutral medium ( $\mathrm{pH}-7.0)$ can be claimed to have good solubility and exhibit good fluorescence in that medium. Dissolution of freeze-dried samples in distilled water $\left(1 \mathrm{mg} \mathrm{ml}^{-1}\right)$ resulted in a transparent and amber-colored solution which implies that the CNPs were hydrophilic (Wu et al., 2017). The highest fluorescence intensity of CNPs was observed at an excitation wavelength of $294 \mathrm{~nm}$ because more number of particles was excited at this wavelength. The hydroxyl groups of lactose molecules added on the surface of CNPs resulted in a series of emissive traps between $\pi-\pi^{*}$ transitions (Peng and Travas-Sejdic, 2009) that corresponds to the shoulder peak (274 $\mathrm{nm}$ ) of UV spectrum depicted in Fig. 1A. The fluorescence emission is a function of particle size wherein the smaller particles emit shorter wavelength near the blue emission area and the larger particles emit longer wavelength near the red emission area. (Walkey et al., 2010). The prolonged heat treatment in the presence of orthophosphoric acid produced smaller particle sizes and stabilized the distribution of surface energy traps which made the CNPs fluoresce in blue color. From TEM and FPS results, the nanosized CNPs with blue fluorescence and excellent dispersion showed complete solubility in water.

The hydrophilic CNPs are advantageous for biological applications; however, their release into the soil as a waste shall be a threat to the growth of existing plants and germination of seeds. Hence, the phytotoxic evaluation of nanomaterials through hydroponic and seedling emergence techniques is important. It was noted that the CNPs showed minimal inhibition towards the growth of scallions at the highest concentration of 1 $\mathrm{mg} \mathrm{ml}$ (Fig. 4A) and was similar to the growth observed during normal conditions. When Indian pearl millets were exposed to CNPs, normal germination rate was observed between the concentration ranges of $0.2-0.6 \mathrm{mg} \mathrm{ml}^{-1}$ and there was no inhibition in the growth of emerging shoots and roots (Fig. 4B). These results corroborates with the earlier studies that assessed the phytotoxic nature of carbon nanomaterial. Hao et al. (2019) and Liu et al. (2015) reported the phytotoxic concentration of carbon based nanoparticles against the growth of rice seedlings to be 0.15 and $0.1 \mathrm{mg} \mathrm{ml}^{-1}$, respectively. In both studies, the reported phytotoxic concentration reduced the length of roots and shoots by at least $50 \%$. In another set of studies conducted by Hu and Zhou (2014) and Chen et al. (2017), graphene oxide nanoparticles inhibited the germination of wheat at $0.2 \mathrm{mg} \mathrm{ml}^{-1}$ and $\geq 0.4 \mathrm{mg} \mathrm{ml}^{-1}$, respectively. Similarly, Anjum et al. (2013) and Raigond et al. (2017) also reported retarded growth of $V$. faba and potato after treatment with water-soluble graphene oxide nanoparticles and zinc nanoparticles at $1.6 \mathrm{mg} \mathrm{ml}^{-1}$ and $500 \mathrm{ppm}$, respectively, due to the induction of oxidative stress.

The cumulative retardation effects of gold and silver nanoparticles in various plants due to severe accumulation were discussed by Dykman and Shchyogolev (2018) revealing the toxic nature of metal nanoparticles. Yet other researchers have reported the positive effect of carbon nanomaterial at lower concentrations but the results varied at high concentrations. Parvin Begum and Bunshi Fugetsu (2012) revealed the nonphytotoxic concentration of carbon nano-tubes against red spinach to be $<0.5 \mathrm{mg} \mathrm{ml}^{-1}$ whereas high concentration of $1 \mathrm{mg} \mathrm{m}^{-1}$ retarded the plant growth and altered the leaf color. Haghighi and Teixeira Da Silva (2014) observed extremely opposite results on the effects of carbon nanotubes (CNTs) on four different seeds and their respective seedlings. CNTs $\left(0.04 \mathrm{mg} \mathrm{ml}^{-1}\right)$ exhibited $<5 \%$ toxicity against tomato and turnip seeds whereas the germination of radish and onion seeds was $86 \%$ and $24 \%$, respectively.

All these studies followed the procedure to determine the potential of seed germination and analyze internalization of carbon nanomaterials. In this study, CNPs dilutions were directly used to the growing plants and it showed no change in growth and cell viability. To confirm penetration and internalization of CNPs into the roots, scallions' rinds were isolated from the exposed root region and observed under a fluorescent microscope. Since the fluorescent spectrum study confirmed blue emission, the U-MWB bandpass excitation filter that transmits only blue excitation waves and a barrier filter BA500 that allows only blue emission were selected to visualize the rinds. Isolated scallion rinds observed under a fluorescent microscope (Fig. 4C) displayed blue fluorescence throughout the rind which indicated that blue fluorescent CNPs penetrated the plant cells and enhanced water uptake, thereby inducing the growth of scallion shoots. In some studies, similar internalization and accumulation of carbon nanoparticles have been reported in various parts of the plant system wherein most of the carbon nanomaterials were observed in the roots, and the penetration level was dependent on the size of nanomaterials (Borisev et al., 2016; Kumar et al., 2018; Li et al., 2016; Verma et al., 2019). On comparing the results, it can be inferred that exposure of blue fluorescent CNPs neither inhibited seed germination nor retarded the growth of scallions, which implies that they were non-phytotoxic.

The cytotoxic level of CNPs was estimated against healthy cell lines by MTT assay. The survival rate of the cells exposed to CNPs was between $95-100 \%$ for $24 \mathrm{hr}$ incubation period (Fig. 5A). The results confirmed that CNPs showed minimum toxicity against MCF10a cell lines even at higher concentrations $\left(1.0 \mathrm{mg} \mathrm{ml}^{-1}\right)$. The microscopic image of MCF10a cell lines exposed to CNPs at $1.0 \mathrm{mg} \mathrm{ml}^{-1}$, as depicted in Fig. 5B, showed that the CNPs caused slight shrinkage, however, no breakage or damage was caused to cell lines. The cytotoxic studies of fluorescent CNPs synthesized from tea (Hsu et al., 2013) and ginger (Li et al., 2014) showed $<5 \%$ toxicity against MCF10a cell lines at a concentration of 0.27 and $1.4 \mathrm{mg} \mathrm{m}^{-1}$, respectively. From these results, it can be suggested that a toxicity value of $<5 \%$ exhibited by CNPs is considered to be noncytotoxic and compatible for MCF10a cell lines.

Internalization of molecules by cells is performed by endocytic pathways such as pinocytosis, phagocytosis and 
clathrin-mediated and caveolin-mediated endocytosis. Pinocytosis engulfs particle size equivalent to $100 \mathrm{~nm}$ whereas phagocytosis engulfs particle size larger than $0.5 \mu \mathrm{m}$. Nanoparticles upto $50 \mathrm{~nm}$ penetrate via caveolin-mediated endocytosis whereas $50-100 \mathrm{~nm}$ size nanoparticles penetrate via clathrin-mediated endocytosis (Villanueva-Flores et al., 2020). In this study, the size of synthesized CNPs ranged between 10-50 $\mathrm{nm}$, the internalization mechanism shall be attributed to caveolinmediated endocytosis. If the CNPs penetrate through caveolinmediated endocytosis mechanism, the CNPs are either degraded by endolysosomes resulting in non-cytotoxic effect or their accumulation in endosomes leading to cytotoxicity (Walkey et al., 2010). Since the results showed non-cytotoxic behavior, it shall be attributed to CNP degradation by the endolysosomes. The water-soluble blue fluorescent CNPs synthesized from lactose seem to be compatible for MCF10a cell line, plant growth and seed germination, hence, it can be considered as a safe nanomaterial for agriculture and biomedical applications.

\section{Acknowledgments}

I would like to thank Vel Tech High Tech Dr. Rangarajan Dr. Sakunthala Engineering College and SSN College of Engineering for providing support for this research work. My sincerity shall be to CLRI, Adyar to perform some of the analytical methods in this work.

\section{References}

Anirudh, S. and D. Joydeep: Small molecules derived carbon dots: Synthesis and applications in sensing, catalysis, imaging and biomedicine. J. Nanobiotechnol., 17, 92 (2019).

Anjum, N.A., N. Singh, M.K. Singh, Z.A. Shah, A.C. Duarte, E. Pereira and I. Ahmad: Single-bilayer graphene oxide sheet tolerance and glutathione redox system significance assessment in faba bean (Vicia faba L.). J. Nanopart. Res., 15, 1770 (2013).

Begum, P. and B. Fugetsu: Phytotoxicity of multi-walled carbon nanotubes on red spinach (Amaranthus tricolor $\mathrm{L}$ ) and the role of ascorbic acid as an antioxidant. J. Hazard. Mater., 243, 212-222 (2012).

Borišev, M., I. Borišev, M. Župunski, D. Arsenov, S. Pajević, Ž. Ćurčić, J. Vasin and A. Djordjevic: Drought impact is alleviated in sugar beets (Beta vulgaris L.) by foliar application of fullerol nanoparticles. PLOS ONE, 11, e0166248 (2016).

Chen, L., C. Wang, H. Li, X. Qu, S.T. Yang and X.L. Chang: Bioaccumulation and toxicity of 13C-skeleton labeled graphene oxide in wheat. Environ. Sci. Technol., 51, 10146-10153 (2017).

Choi, J. and N. Wang: Nano-particles in biomedical applications and their safety concerns. In: Biomedical Engineering - From Theory to Applications (Ed.: Reza Fazel-Rezai). Intech Open, pp. 299-314 (2011).

Curtis, R.W.: Studies on response of bean seedlings and corn roots to malformin. Plant Physiol., 36, 37-43 (1961).

Dykman, L. and S. Shchyogolev: The effect of gold and silver nanoparticles on plant growth and development. In: Metal Nanoparticles: Properties, Synthesis and Applications (Ed.: Y. Saylor and V. Irby). Nova Science Publishers, Inc., pp.1-138 (2018).

Gatoo, M., S. Naseem, M.Y. Arfat, A.M. Dar, K. Qasim and S. Zubair: Physico-chemical properties of nanomaterials: Implication in associated toxic manifestations. BioMed. Res. Int., 2014, 498420 (2014).

Haghighi, M. and J.A. Teixeira Da Silva: The effect of carbon nanotubes on the seed germination and seedling growth of four vegetable species. J. Crop Sci. Biotechnol., 17, 201-208 (2014).

Hao, Y., B. Xu, C. Ma, J. Shang, W. Gu, W. Li, T. Hou, Y. Xiang, W. Cao, B. Xing and Y. Rui: Synthesis of novel mesoporous carbon nanoparticles and their phytotoxicity to rice (Oryza sativa L.). J. Saudi Chem. Soc., 23, 75-82 (2019).

Hsu, P., P. Chen, C. Ou, H. Chang and H. Chang: Extremely high inhibition activity of photoluminescent carbon nanodots toward cancer cells. J. Mater. Chem. B, 1, 1774-1781 (2013).

$\mathrm{Hu}, \mathrm{X}$. and Q. Zhou: Novel hydrated graphene ribbon unexpectedly promotes aged seed germination and root differentiation. Sci. Rep., 4, 3782 (2014).

Kim, D., Y. Choi, E. Shin, Y. Jung and B. Kim: Sweet nanodot for biomedical imaging: Carbon dot derived from xylitol. RSC Adv., 4, 23210-23213(2014).

Kokorina, A.A., E.S. Prikhozhdenko, G.B. Sukhorukov, A.V. Sapelkin and I.Y. Goryacheva: Luminescent carbon nanoparticles: Synthesis, methods of investigation applications. Russ. Chem. Rev., 86, 1157-1171 (2017).

Kumar, A., A. Singh, M. Panigrahy, P.K. Sahoo and K.C. Panigrahi: Carbon nanoparticles influence photomorphogenesis and flowering time in Arabidopsis thaliana. Plant Cell Rep., 37, 901-912(2018).

Lamer, V.K. and R.H. Dinegar: Theory, production and mechanism of formation of monodispersed hydrosols. J. Am. Chem. Soc., 72, 4847-4854 (1950).

Li, W., Y. Zheng, H. Zhang, Z. Liu, W. Su, S. Chen, Y. Liu, J. Zhuang and B. Lei: Phytotoxicity, uptake and translocation of fluorescent carbon dots in mung bean plants. ACS Appl. Mater. Interfaces, 8, 19939-19945 (2016).

Li, Y., X. Zhong, A. Rider, S. Furman and K. Ostrikov: Fast, energyefficient synthesis of luminescent carbon quantum dots. Green Chem., 16, 2566-2570 (2014)

Liu, H., Z. Li, Y. Sun, X. Geng, Y. Hu, H. Meng, J. Ge and L. Qu: Synthesis of luminescent carbon dots with ultrahigh quantum yield and inherent folate receptor-positive cancer cell targetability. Sci. Rep., 8, 1086 (2018).

Liu, S., H. Wei, Z. Li, S. Li, H. Yan, Y. He and Z. Tian: Effects of graphene on germination and seedling morphology in rice. J. Nanosci. Nanotechnol., 15, 2695-2701(2015).

Mora, P. and J. Wood: Synthetic Polysaccharides. I. Polycondensation of Glucose. J. Am. Chem. Soc., 80, 685-692 (1958).

Mosmann, T.: Rapid colorimetric assay for cellular growth and survival: Application to proliferation and cytotoxicity assays. J. Immunol. Methods, 65, 55-63 (1983).

Peng, $\mathrm{H}$. and J. Travas-Sejdic: Simple aqueous solution route to luminescent carbogenic dots from carbohydrates. Chem. Mater., 21,5563-5565 (2009).

Pengli, Z., L. Xiuhua, S. Zhi-Gang, G. Yuhan and H. Hua: A review on syntheses, properties, characterization and bioanalytical applications of fluorescent carbon dots. Microchim. Acta, 183, 519542 (2016).

Raigond, P., B. Raigond, B. Kaundal, B. Singh, A. Joshi and S. Dutt: Effect of zinc nanoparticles on antioxidative system of potato plants. J. Environ. Biol., 38, 435-439 (2017).

Sahu, S., B. Behera, T. Maiti and S. Mohapatra: Simple one-step synthesis of highly luminescent carbon dots from orange juice: Application as excellent bio-imaging agents. Chem. Commun., 48, 8835-8837 (2012).

Sezer, D. and E. Cevher: Biopolymers as wound healing materials: 
Challenges and new strategies. In: Biomaterials Applications for Nanomedicine (Ed.: Rosario Pignatello). Intech, pp. 383-414 (2011).

Shi, H., J. Wei, L. Qiang and X. Chen: Fluorescent carbon dots for bioimaging and biosensing applications. J. Biomed. Nanotechnol., 10, 2677-2699 (2014).

Sk, M., A. Jaiswal, A. Pau, S. Ghosh and A. Chattopadhyay: Presence of amorphous carbon nanoparticles in food caramels. Sci. Rep., 2, $383(2012)$

Song, Y., S. Zhu and B. Yang: Bioimaging based on fluorescent carbon dots. RSCAdv., 4, 27184 (2014)

Verma, S.K., A.K. Das, S. Gantait, V. Kumar and E. Gurel:Applications of carbon nanomaterials in the plant system: A perspective view on the pros and cons. Sci. Total Environ., 667, 485-499 (2019).

Villanueva-Flores, F., A. Castro-Lugo, O. Ramírez and L. Palomares: Understanding cellular interactions with nanomaterials: Towards a rational design of medical nanodevices. Nanotechnology, 31, $132002(2020)$

Walkey, C., E.A. Sykes and W.C.W. Chan: Application of semiconductor and metal nanostructures in biology and medicine. Hematol. Am. Soc. Hematol. Educ. Prog., 2009, 701-707 (2009).

Wu, L., X. Cai, K. Nelson, W. Xing, J. Xia, R. Zhang, A.J. Stacy, M. Luderer, G.M. Lanza, L.V. Wang, B. Shen and D.A. Pan: Green synthesis of carbon nanoparticle from honey for real time photoacoustic imaging. Nano Res., 6, 312-325 (2013).

Wu, Z., Z. Liub and Y. Yuan: Carbon Dots: Materials, synthesis, properties and approaches to long-wavelength and multicolor emission. J. Mater. Chem., B, 5, 3794-3809 (2017).

Zhang, X., M. Jiang, N. Niu, Z. Chen, S. Li, S. Liu and J. Li: Naturalproduct-derived carbon dots: From natural products to functional materials. Chem. Sus. Chem., 11, 11-24 (2018).

Zhang, Y., Y. Wang, X. Feng, F. Zhang, Y. Yang and X. Liu: Effect of reaction temperature on structure and fluorescence properties of nitrogendoped carbon dots. Appl. Surf. Sci., 387, 1236-1246 (2016).

Zhang, Z., X. Wang, X. Zhang, P. Yu, L. Geng and S. Shi: Preparation of fluorescent carbon dots and their interaction with humantransferrin. Mater. Res. Bull., 99, 225-331 (2018). 